\title{
Building a Case for Withaferin A as a Treatment for FTD/ALS Syndromes
}

\section{David R. Borchelt ${ }^{1}$ (D)}

Accepted: 13 December 2020 / Published online: 4 January 2021

(C) The American Society for Experimental NeuroTherapeutics, Inc. 2021

Withaferin A (WFA) is a natural compound produced by Withania somnifera, which has been used as a traditional medicinal herb for centuries (reviewed in [1]). Over the past few decades, investigators have begun to delve into the wealth of natural compounds to find new therapeutic approaches for a host of diseases that still have few therapeutic options, including neurodegenerative diseases. WFA, a steroid lactone, is an example of a natural compound that has been investigated as a potential therapy for several disorders including certain types of cancers, metabolic disorders, inflammatory conditions, and neurodegenerative disorders (reviewed in [2]).

In the current issue, Kumar and colleagues examined the potential for WFA to mitigate neurologic disease associated with abnormal TDP43 [3]. The present study follows up on prior work from this group that has been investigating the role of NFKB signaling in the pathogenesis of amyotrophic lateral sclerosis [4-6]. One of the activities that has been credited to WFA is to inhibit NFKB signaling to reduce neuroinflammation (reviewed in [7]). In prior work, TDP43 with mutations associated with amyotrophic lateral sclerosis and frontotemporal dementia was shown to interact with $\mathrm{NFKB}$ and aberrantly activate neuroinflammatory pathways [5]. In the study in the current issue, the authors confirm the ability of WFA to inhibit inflammatory pathways regulated by NF $k B$. They then treat mice that express human TDP43 with the G348C mutation found in FTD/ALS with WFA at $5 \mathrm{mg} / \mathrm{kg}$ by i.p. injection every other day. The G348C model develops cognitive deficits at about 12 months of age, with motor deficits developing at later ages. The authors initiated treatment at 10 months of age, which is the approximate age that the mice

David R. Borchelt

drb1@ufl.edu

1 Department of Neuroscience, University of Florida College of Medicine, McKnight Brain Institute, Center for Translational Research in Neurodegenerative Disease, University of Florida, Gainesville, FL 32610, USA will have begun to develop abnormal TDP43 pathology in CNS neurons. Mutant TDP43 mice treated in this way were found to have reduced cytoplasmic TDP43 inclusion pathology and improved performance in a passive avoidance cognitive test. This report appears to be the first to demonstrate that WFA could enhance cognition in neurodegenerative models.

WFA has been reported to exhibit numerous activities that could be beneficial in neurodegenerative disease [8]. Consistent with other studies of WFA activities, Kumar and colleagues report that WFA treatment induced autophagy in mutant TDP43 mice. Whether this activity was responsible for the reduction in inclusion pathology is uncertain, but the data support the idea that WFA could produce multiple beneficial effects.

WFA is widely available as a nutraceutical that is sold as Ashwaganda extract that is taken orally. It is important to note that the concentration of WFA within these unregulated extracts may vary considerably. Although WFA appears to be orally bioavailable [9], there is no information on the oral dose that would be required to achieve the same positive effects seen in mice injected intraperitoneally. Moreover, there has not been an assessment of safety for WFA in ALS/FTD patients; however, toxicological studies of WFA and other Ashwaganda compounds indicate low toxicity [8]. Plant extracts may induce allergic reactions, or other unpleasant gastrointestinal reactions, that could be problematic. The promising results described by Kumar et al. in the current issue, and in other prior work by this group $[4,5]$, may provide motivation for further study of this molecule in ALS/FTD patients.

Supplementary Information The online version contains supplementary material available at https://doi.org/10.1007/s13311-020-00991-7.

\section{References}

1. Mukherjee PK, Banerjee S, Biswas S, Das B, Kar A, Katiyar CK. Withania somnifera (L.) Dunal - Modern perspectives of an ancient Rasayana from Ayurveda. J Ethnopharmacol. Ireland; 2021; 264: 113157. 
2. Behl T, Sharma A, Sharma L, Sehgal A, Zengin G, Brata R, et al. Exploring the Multifaceted Therapeutic Potential of Withaferin A and Its Derivatives. Biomedicines; 2020; 8: E571. Switzerland

3. Kumar S, Phaneuf D, Julien J-P. Withaferin-A Treatment Alleviates TAR DNA-Binding Protein-43 Pathology and Improves Cognitive Function in a Mouse Model of FTLD. Neurother J Am Soc Exp Neurother. United States; 2020;

4. Patel P, Julien J-P, Kriz J. Early-stage treatment with Withaferin A reduces levels of misfolded superoxide dismutase 1 and extends lifespan in a mouse model of amyotrophic lateral sclerosis. Neurother J Am Soc Exp Neurother. 2015; 12:217-33.

5. Swarup V, Phaneuf D, Dupré N, Petri S, Strong M, Kriz J, et al. Deregulation of TDP-43 in amyotrophic lateral sclerosis triggers nuclear factor $\mathrm{kB}$-mediated pathogenic pathways. J Exp Med. 2011; 208:2429-47.

6. Dutta K, Patel P, Rahimian R, Phaneuf D, Julien J-P. Withania somnifera Reverses Transactive Response DNA Binding Protein
43 Proteinopathy in a Mouse Model of Amyotrophic Lateral Sclerosis/Frontotemporal Lobar Degeneration. Neurother J Am Soc Exp Neurother. 2017; 14:447-62.

7. Logie E, Vanden Berghe W. Tackling Chronic Inflammation with Withanolide Phytochemicals-A Withaferin a Perspective. Antioxidants (Basel, Switzerland). 2020; 9:1107

8. Zahiruddin S, Basist P, Parveen A, Parveen R, Khan W, Gaurav, et al. Ashwagandha in brain disorders: A review of recent developments. J Ethnopharmacol. Ireland; 2020; 257:112876.

9. Dai T, Jiang W, Guo Z, Wang Z, Huang M, Zhong G, et al. Studies on oral bioavailability and first-pass metabolism of withaferin $\mathrm{A}$ in rats using LC-MS/MS and Q-TRAP. Biomed Chromatogr England; 2019; 33:e4573.

Publisher's Note Springer Nature remains neutral with regard to jurisdictional claims in published maps and institutional affiliations. 